\title{
Malrotation Anomaly Left Lower Quadrant Appendicitis
}

\author{
Yunus Dönder', Mehmet Baykan², Betül Güney ${ }^{3}$ \\ 'Clinic of General Surgery, Kilis State Hospital, Kilis, Turkey \\ ${ }^{2}$ Clinic of General Surgery, Private Melikgazi Hospital, Kayseri, Turkey \\ ${ }^{3}$ Clinic of General Radyology, Private Melikgazi Hospital, Kayseri, Turkey
}

Cite this article as: Dönder Y, Baykan M, Güney B. Malrotation Anomaly Left Lower Quadrant Appendicitis. J Emerg Med Case Rep 2018; 9: 44-6.

\begin{abstract}
Introduction: Acute appendicitis is one of the most commonly diagnosed surgical diseases in children with emergency abdominal pain. Atypical localized appendicitis is rare and difficult so we can see delay in diagnosis. Malrotation anomaly is also a cause of left-sided appendicitis. Herein, we present a rare case of acute appendicitis in the left lower quadrant with malrotation anomaly.

Case Report: A 12-year-old male patient was admitted to the emergency department due to abdominal pain since 2 days. We discovered that the abdominal pain was initially widespread in the abdomen and gradually dispersed to the lower left side. Medical examination and radiological assessment were conducted, and a diagnosis of acute appendicitis in left lower quadrant was made. The patient subsequently underwent surgery and was discharged on the second postoperative day.

Conclusion: There are many causes of left lower quadrant pain in the abdomen. In cases with suspected findings on physical examination, imaging methods can be used to diagnose rare incidents.
\end{abstract}

Keywords: Malrotation, acute appendicitis, left lower quadrant pain

Received: 11.08.2017 Accepted: 18.01.2018

\section{Introduction}

Acute appendicitis is a common surgical condition (1). It may be caused by various conditions including gastritis, gastroenteritis, cholecystitis, pyelonephritis, and diverticulitis due to the location of the pain and similar diseases. Some diagnoses may become more difficult with the presence of accompanying anatomic anomalies (2). Appendicitis can occur in the left lower quadrant in patients with intestinal malrotation, situs inversus totalis, mobile appendix, and chronic appendicitis. These conditions are rarely encountered and are thus not initially considered in the differential diagnosis of left lower quadrant pain. This can lead to delayed diagnosis. Herein, we aimed to present a rare case of appendicitis in the left lower quadrant due to malrotation.

\section{Case Report}

A 12-year-old male patient was admitted to the emergency department due to abdominal pain since 2 days. We discovered that the abdominal pain was initially widespread in the abdomen and gradually dispersed to the lower left side. There was no nausea, vomiting, and diarrhea in the patient anamnesis. Physical examination was sensitivity and rebound was positive in the left lower quadrant of abdomen. Laboratory findings revealed leukocytosis in the analysis of the whole blood count (18,000/ $\mathrm{mm}^{3}$ ), and his serum biochemical values were normal. Leukocytes and erythrocytes were absent in the urine test. The patient underwent an abdominal ultrasound for the diagnosis of abdominal pain, and as a result, a "target sign" was seen in the left lower

Address for Correspondence:

Yunus Dönder, Clinic of General Surgery, Kilis State Hospital, Kilis, Turkey

E-mail:ydonder@hotmail.com

oCopyright 2018 by Emergency Physicians Association of Turkey - Available online at www.jemcr.org 


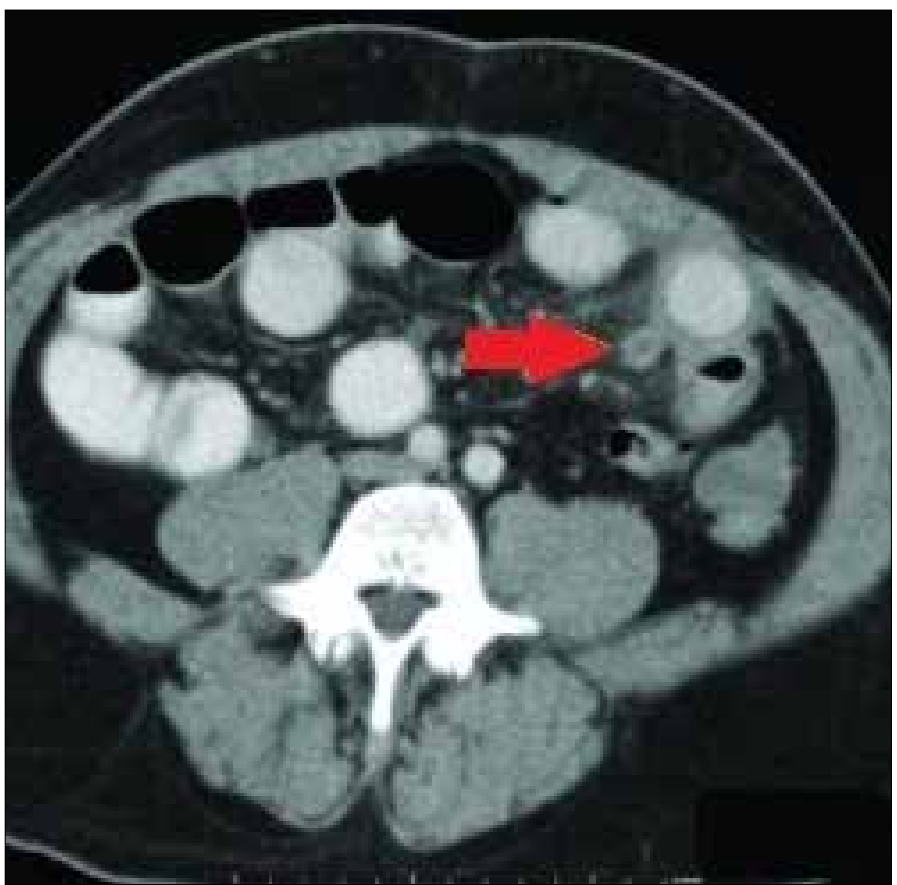

FIGURE 1. Axial computed tomography scan showing left-sided appendicitis (red arrow).

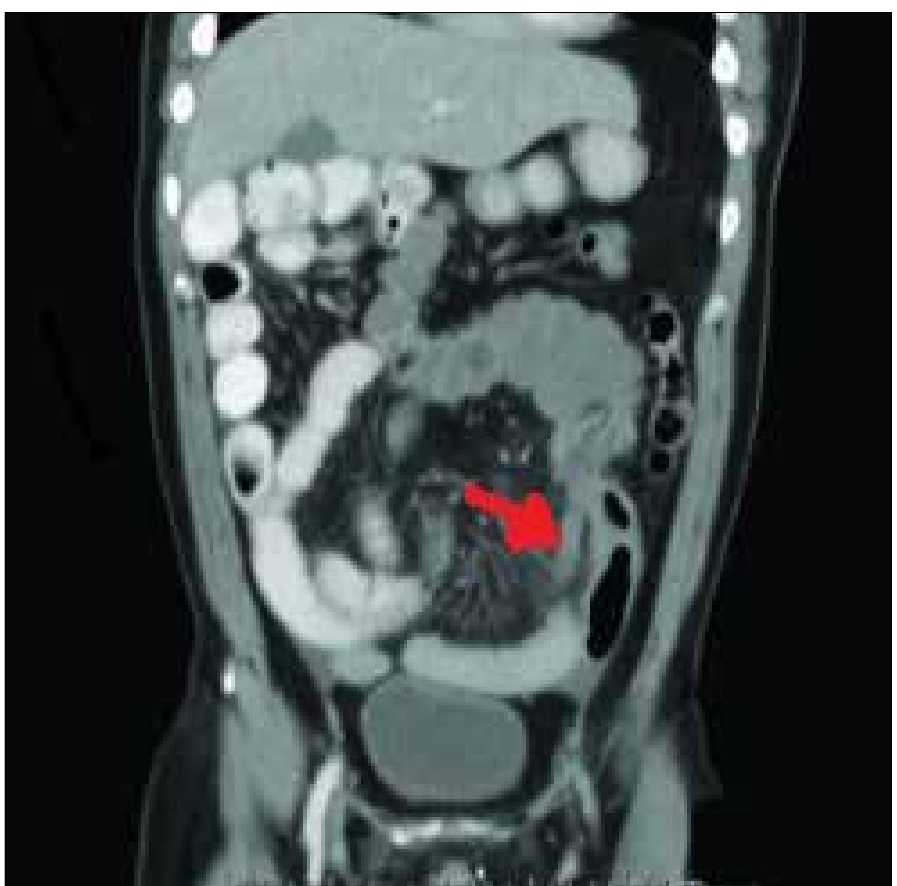

FIGURE 2. Coronal computed tomography scan showing leftsided inflamed acute appendicitis (red arrow).

quadrant of the abdomen; however, the diagnosis could not be confirmed, thereby necessitating computed tomographic evaluation. Computed tomography (CT) was performed, and $16 \mathrm{~mm}$ blind ending luminal structure near the bladder was seen in the left lower site of abdomen. Lymphadenopathy was seen in this region. Malrotation and acute appendicitis were interpreted (Figures 1 and 2). After pro-

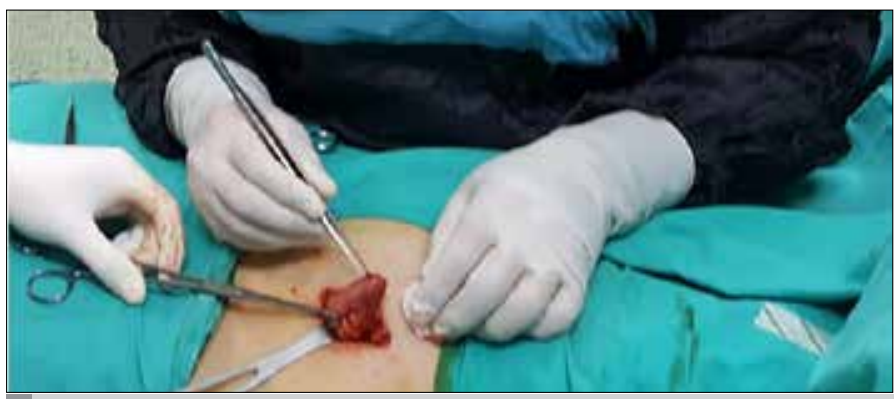

FIGURE 3. Operation images of left-sided appendicitis.

viding verbal and written informed consent, the patient underwent appendectomy, where in a left McBurney incision was made (Figure 3). The patient was diagnosed with acute perforated appendicitis by physical examination and received liquid diet on the first postoperative day. The patient was discharged with good general conditions on the second postoperative day.

\section{Discussion}

Patients presenting to the hospital with left lower quadrant pain in the abdomen. In this patient group, the sigmoid colon diverticulum, urinary tract pathologies, gynecological causes, invagination, volvulus, renal colic, abdominal aortic aneurysm, epididymitis, and testicular torsion usually considered. Acute appendicitis in the left lower quadrant may also occur in patients with malrotation and situs inversus totalis.

However, if this condition is not known in advance, it may cause delayed diagnosis, leading to increased morbidity and mortality. In a study conducted by Collins et al. (3), the incidence of appendicitis with left lower quadrant pain was reported as 0.04\%, and 0.024\% of cases were associated with malrotation of the intraabdominal organs and $0.016 \%$ with situs inversus totalis. In our case, malrotation was detected by ultrasound and CT.

Midgut malrotation is associated with an abnormal rotation of the primitive intestine around the first mesenteric artery axis during the first 10 weeks of fetal life (1). The majority of these cases (85\%) occur in the first month of life with unclear abdominal pain, bloating, vomiting, or obstruction. A small proportion, with an incidence of $0.1 \%-0.5 \%$, progresses to adulthood and remains asymptomatic (4). In this case patient was 12-year-old child, it is in accordance with the literature. The use of ultrasound for malrotation seems to be advantageous for children because it does not lead to an X-ray effect, and it also provides a correct diagnosis. Directional change of the upper mesenteric vessels is diagnostic. In a study of 23 patients by Zhou et al. (5), the sensitivity of ultrasonography (100\%) and the specificity (97.8\%) of malrotation were reported. However, Orzechet al. (6) reported a positive predictive value of $42.1 \%$ and a negative predictive value of $96.3 \%$ in the study of 211 patients with malrotation on ultrasound. Ultrasound findings can be used for diagnosing malrotation, but further imaging is required if they suggest abnormal bowel fixation (6). In our case, malrotation was suspected on ultrasound and its diagnosis was confirmed by CT. 


\section{Conclusion}

There are many causes of left lower quadrant pain in the abdomen. In cases with suspected findings on physical examination, imaging methods can be used to diagnose rare incidents. Thus, it is possible to achieve more pleasant results in terms of patient and physician.

Informed Consent: Written and verbal informed consent form taken from patient and patient's parents.

Peer-review: Externallypeer-reviewed.

Author Contributions: Concept - Y.D.; Design - Y.D.; Supervision - Y.D.; Resources - M.B.; Materials - M.B.; Data Collection and/or Processing - B.G.; Analysis and/or Interpretation - B.G.; Literature Search - Y.D.; Writing Manuscript - Y.D.; Critical Review - M.B.

Conflict of Interest: The authors have no conflict of interest to declare.

Financial Disclosure: The authors declared that this study has received no financial support.

\section{References}

1. Akbulut S, Ulku A, Senol A, Tas M, Yagmur Y. Left-sided appendicitis: review of 95 published cases and a case report. World J Gastroenterol 2010; 16: 5598-602. [CrossRef]

2. Hanna T, Akoh JA. Acute presentation of intestinal malrotation in adults: a report of two cases. Ann R Coll Surg Engl 2010; 92:W15-8. [CrossRef]

3. Collins DC. 71,000 Human Appendix Specimens. A Final Report, Summarizing Forty Years' Study. Am J Protocol 1963; 14: 265-81.

4. Ben Ely A, Gorelik N, Cohen-Sivan Y, Zissin R, Carpineta L, Osadchy A, et al. Appendicitis in adults with incidental midgut malrotation: CT findings. Clin Radiol 2013; 68: 1212-9. [CrossRef]

5. Zhou LY, Li SR, Wang W, Shan QY, Pan FS, Liu JC, et al. Usefulness of sonography in evaluating children suspected of malrotation: Comparison with an upper gastrointestinal contrast study. J Ultrasound Med 2015; 34: 1825-32. [CrossRef]

6. Orzech N, Navarro OM, Langer JC. Is ultrasonography a good screening test for intestinal malrotation? J Pediatr Surg 2006; 41: 1005-09. [CrossRef] 